\title{
Pulse fidelity in ultra-high-power (petawatt class) laser systems
}

\author{
Colin Danson ${ }^{1,2}$, David Neely ${ }^{3}$, and David Hillier ${ }^{2}$ \\ ${ }^{1}$ Centre for Inertial Fusion Studies (CIFS), Imperial College London, UK \\ ${ }^{2}$ AWE plc, Aldermaston, UK \\ ${ }^{3}$ Central Laser Facility, STFC Rutherford Appleton Laboratory, UK \\ (Received 5 June 2014; accepted 28 August 2014)
}

\begin{abstract}
There are several petawatt-scale laser facilities around the world and the fidelity of the pulses to target is critical in achieving the highest focused intensities and the highest possible contrast. The United Kingdom has three such laser facilities which are currently open for access to the academic community: Orion at AWE, Aldermaston and Vulcan \& Astra-Gemini at the Central Laser Facility (CLF), STFC (Science and Technology Facilities Council) Rutherford Appleton Laboratory (RAL). These facilities represent the two main classes of petawatt facilities: the mixed OPCPA/Nd:glass high-energy systems of Orion and Vulcan and the ultra-short-pulse Ti:Sapphire system of AstraGemini. Many of the techniques used to enhance and control the pulse generation and delivery to target have been pioneered on these facilities. In this paper, we present the system designs which make this possible and discuss the contrast enhancement schemes that have been implemented.
\end{abstract}

Keywords: petawatt laser; contrast; wavefront correction; plasma mirror

\section{Systems description}

The facilities highlighted within this paper are operated at AWE, Aldermaston, UK and the Central Laser Facility (CLF), STFC (Science and Technology Facilities Council) Rutherford Appleton Laboratory (RAL), UK and have the following system parameters:

- Orion is the latest facility to be built in the UK and became operational in April 2013 ${ }^{[1]}$. It is a Nd:glass laser system which combines 10 long-pulse beamlines (500 J, 1 ns @ $351 \mathrm{~nm})$ with two synchronized infrared petawatt beams (500 J in $500 \mathrm{fs})$. Up to $15 \%$ of the Orion beam time is available to the UK academic community.

- HELEN is the Orion predecessor and was operational at AWE for nearly 30 years $^{[2]}$. It had two $527 \mathrm{~nm}$, $500 \mathrm{~J}$ long-pulse (nanosecond) beamlines capability with a synchronized 100 TW short-pulse beam. It closed down in April 2009 to allow resources to be switched to the Orion facility during its commissioning.

Correspondence to: Colin Danson, AWE, Aldermaston, Reading, RG7 4PR, UK. Email: c.danson@imperial.ac.uk
- Vulcan is a high-power Nd:glass academic user facility $^{[3]}$ which has been operational for over 30 years. It enables a broad range of experiments through a flexible geometry ${ }^{[4,5]}$. It has two target areas: one with $6 \times 300 \mathrm{~J}$ (1053 nm @1 ns) long pulses combined with two synchronized short-pulse beams and a separate target area with high-energy petawatt capability (500 J in $500 \mathrm{fs}$ ) synchronized with a single long-pulse beamline.

- Astra-Gemini is a Ti:Sapphire laser system ${ }^{[6]}$ operating at $800 \mathrm{~nm}$ pumped by green pulsed lasers in multi-stage amplifiers. It is operated as an academic user facility. It has two ultra-high-power beamlines delivering $15 \mathrm{~J}$ in $30 \mathrm{fs}$ pulses @ $@ 800 \mathrm{~nm}$, generating focused intensities $>10^{21} \mathrm{~W} \mathrm{~cm}{ }^{-2}$ to target.

\section{Petawatt generation delivering focused intensities of $10^{21} \mathrm{~W} \mathrm{~cm}^{-2}$}

Short-pulse capabilities on all these systems are based on the technique of chirped pulse amplification $(\mathrm{CPA})^{[7]}$, where an ultra-short pulse is stretched, amplified and then recompressed to overcome nonlinear propagation issues primarily 

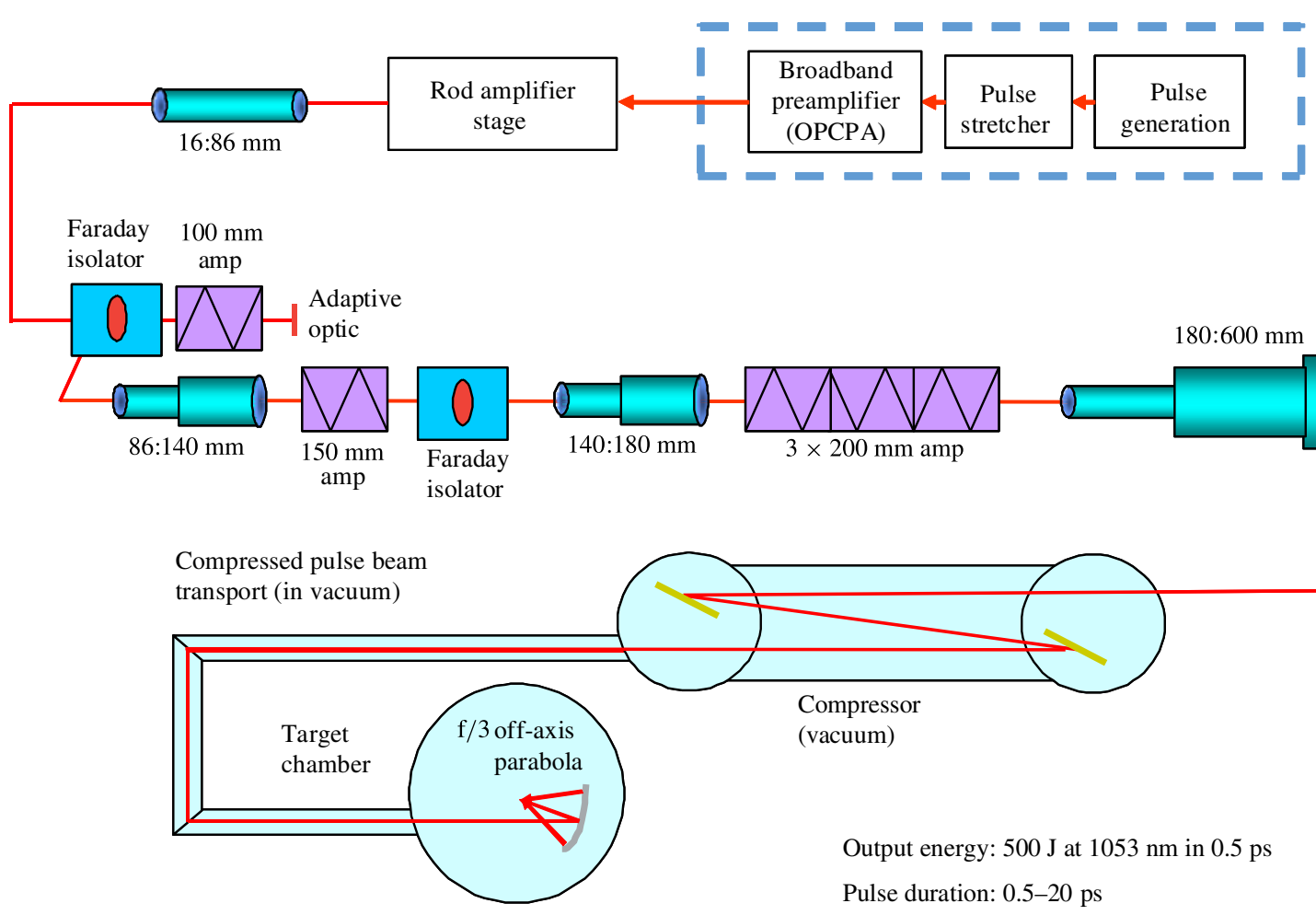

Figure 1. Layout of the Orion/Vulcan petawatt laser systems.

in the glass amplifiers. The first kilojoule class system to be configured to deliver a petawatt was the Nova Facility at LLNL $^{[8]}$. All of the basic building blocks used on later systems were deployed on Nova, including: broadbandwidth pulse generation; optical pulse stretching; pulse amplification; deformable mirrors; pulse compression and reflective focusing.

\subsection{Orion \& Vulcan ultra-high-power delivery}

The Orion and Vulcan short-pulse petawatt beamlines are based on the master oscillator-power amplifier (MOPA) architecture (see Figure 1). A pulse generated in a commercial Ti:Sapphire oscillator is initially stretched in a doublepass Offner stretcher. Its first stages of amplification are in a broadband three-stage optical parametric chirped pulse amplifier (OPCPA $)^{[9,10]}$. Further amplification in mixed glass (phosphate/silicate) takes the energy to the joule level prior to the final amplification in three stages of disc amplifiers (100 $\mathrm{mm}$ \{double-passed\}, 150 and $200 \mathrm{~mm}\{\times 3\}$ aperture). An adaptive optic mirror, a critical component in generating the ultra-high intensities to target, is positioned as the reflecting mirror between the double passes of the $100 \mathrm{~mm}$ disc amplifier. Feedback is provided from a Hartmann sensor positioned in the output diagnostic package.

Following the disc amplifier stages, a beam at the $700 \mathrm{~J}$ level is expanded to $600 \mathrm{~mm}$ in diameter and compressed in a single-pass geometry using a pair of gold-coated diffraction

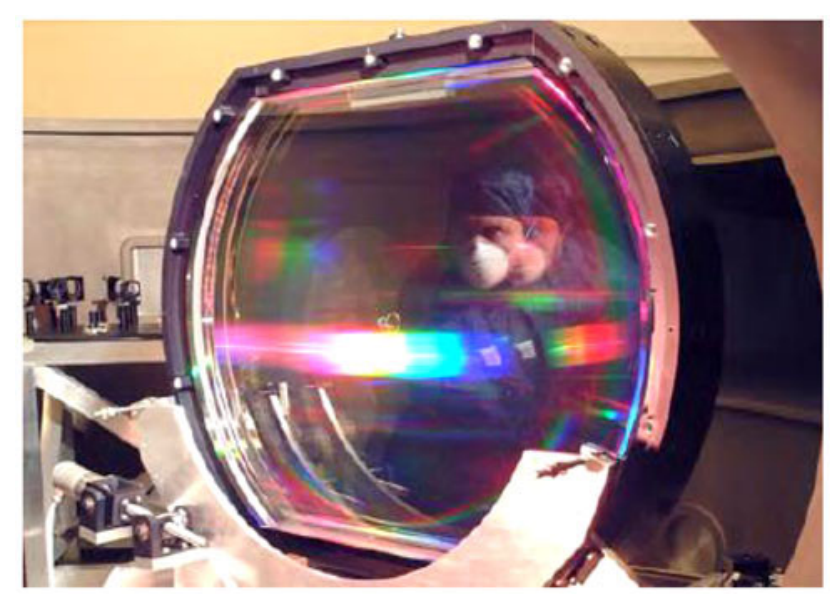

Figure 2. One of the $940 \mathrm{~mm}$ aperture compressor gratings installed on the Vulcan Petawatt beamline.

gratings (with 1480 lines $\mathrm{mm}^{-1}$ ) separated by $13 \mathrm{~m}$ and housed in a large vacuum chamber. The resulting $500 \mathrm{~J}$, 0.5 ps beams are then propagated to target in vacuum and focused using an F/3, $1.8 \mathrm{~m}$ focal length off-axis parabola (OAP).

There is now vast experience and understanding of the generation of petawatt pulses to target, as detailed in $[1,3,11]$. It can be summarized as:

- Deliver enough energy (>500 J) limited by grating damage (see Figure 2 for the large, $940 \mathrm{~mm}$ aperture installed on Vulcan Petawatt) 

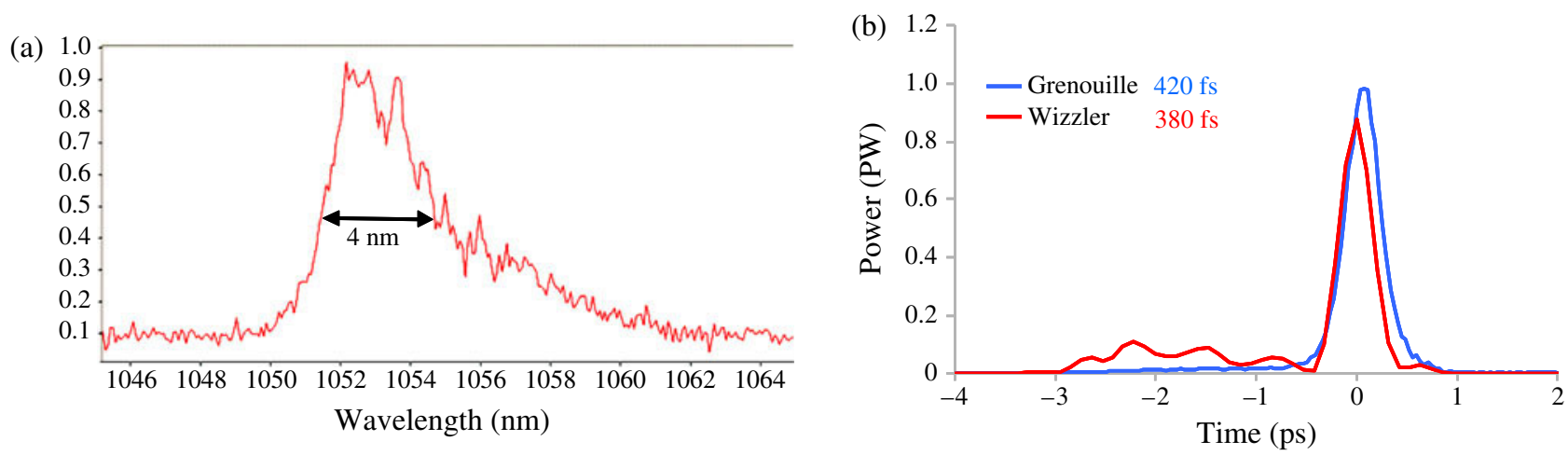

Figure 3. (a) The Orion petawatt output bandwidth. (b) The Orion output temporal profile demonstrating $<500$ fs pulsewidth.
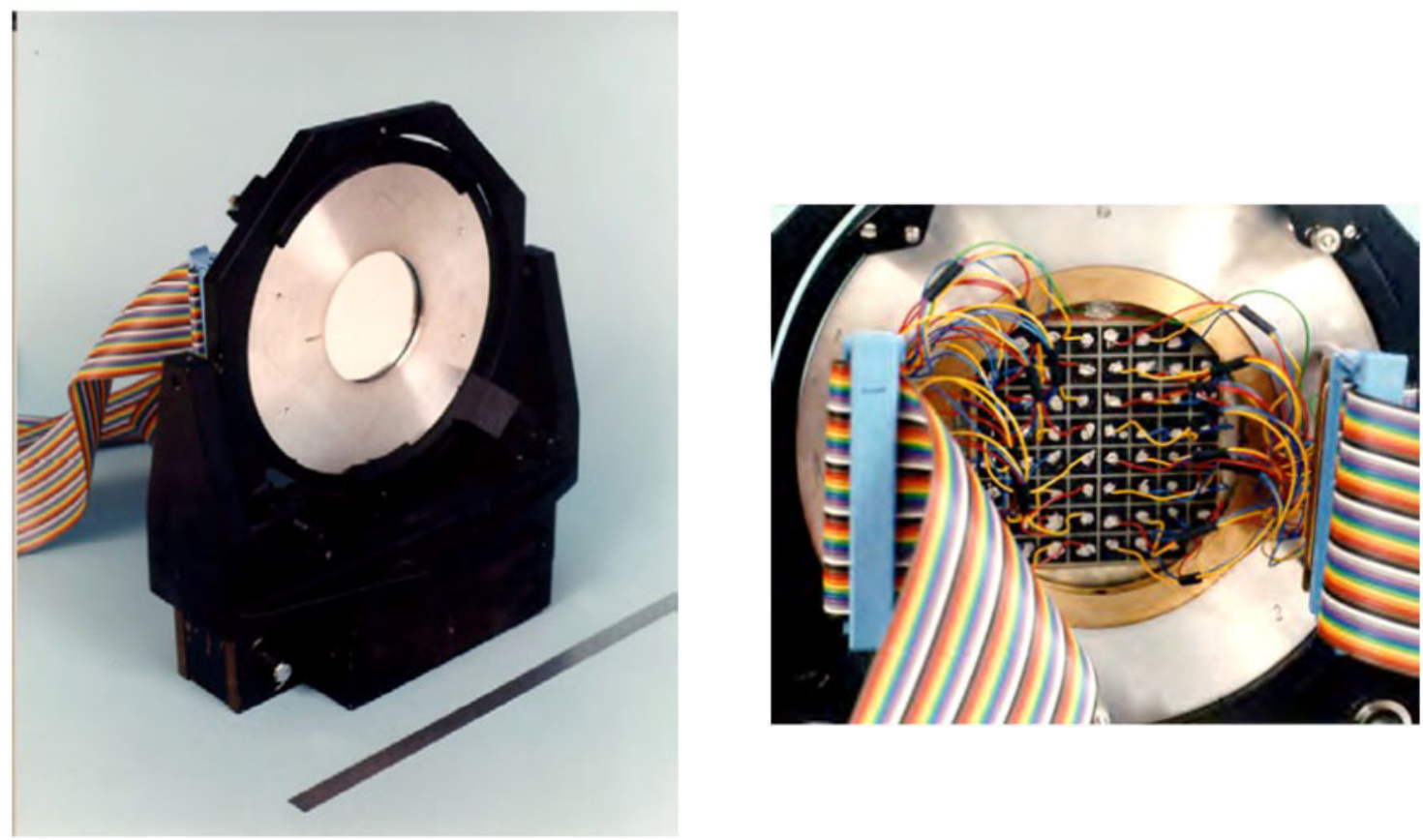

Figure 4. Prototype adaptive mirror as deployed on Vulcan Petawatt.

- Generate sufficient bandwidth ( $>4 \mathrm{~nm}$; see Figure 3(a))

- Optimize the stretcher/compressor for short-pulse delivery $(<500$ fs see Figure 3(b)).

Having generated the petawatt beam, the use of adaptive optics holds the key to achieving the spot size and pulse duration required for irradiances on target $>10^{20} \mathrm{~W} \mathrm{~cm}^{-2}$. In CLF tests, an adaptive optics system achieved a cycle rate of $\sim 8 \mathrm{~Hz}$ with correction to $0.45 \lambda$. At the time that Vulcan Petawatt was being developed there were no commercial solutions to large aperture wavefront correction; therefore, an in-house prototype ' $8 \times 8$ bimorph' mirror was developed at RAL (see Figure 4).

The final output beam to target was found to have residual astigmatism which could be compensated for by optimizing the focal spot using the offset and tilt of the OAP. The spots were first optimized using the Vulcan narrowband CW alignment beam and then optimized at the joule level using rod amplifier shots. Focal spot diameters of $\sim 7 \mu \mathrm{m}$ were generated to target (see Figure 5).

On full-energy shots, the focal spot on target was monitored using X-ray pinhole camera imaging, resulting in focal spot sizes of $\sim 11 \mu \mathrm{m}$ FWHM (the X-ray image is likely to be larger than the optical image due to plasma expansion), giving focused intensities to target on high-energy shots of $\sim 10^{21} \mathrm{~W} \mathrm{~cm}^{-2}$.

On Orion, a commercial wavefront corrector (Imagine Optics/CILAS) could be deployed. With a nominally flat mirror in the deformable mirror location an aberration of $2.5 \lambda$ peakto-valley (mostly astigmatism) could be observed. With the 63 -element monomorph deformable mirror in place this is 


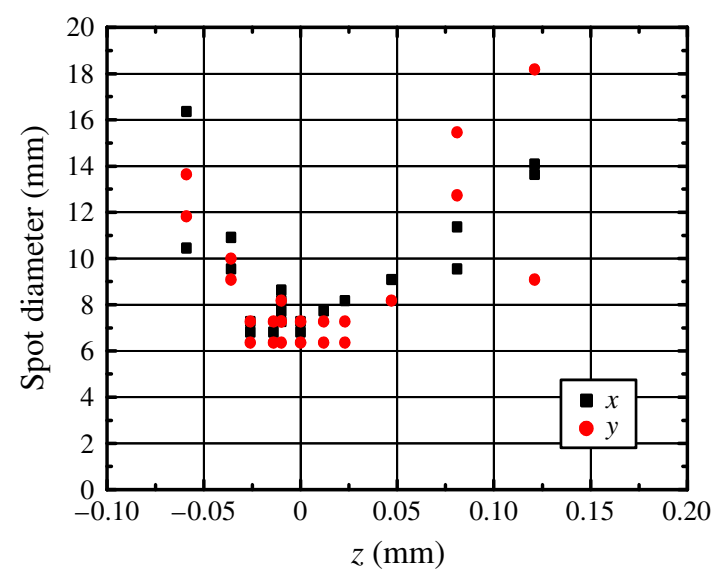

Figure 5. Vulcan Petawatt beam focal spot scan to target using joule-level pulses.

corrected to $<0.5 \lambda$ peak-to-valley. As with Vulcan, the optimization to target is done using the final focusing OAP. The optical images, taken in the output beam diagnostics prior to the compressors and shown in Figures 6(a) and 6(b),

(a)
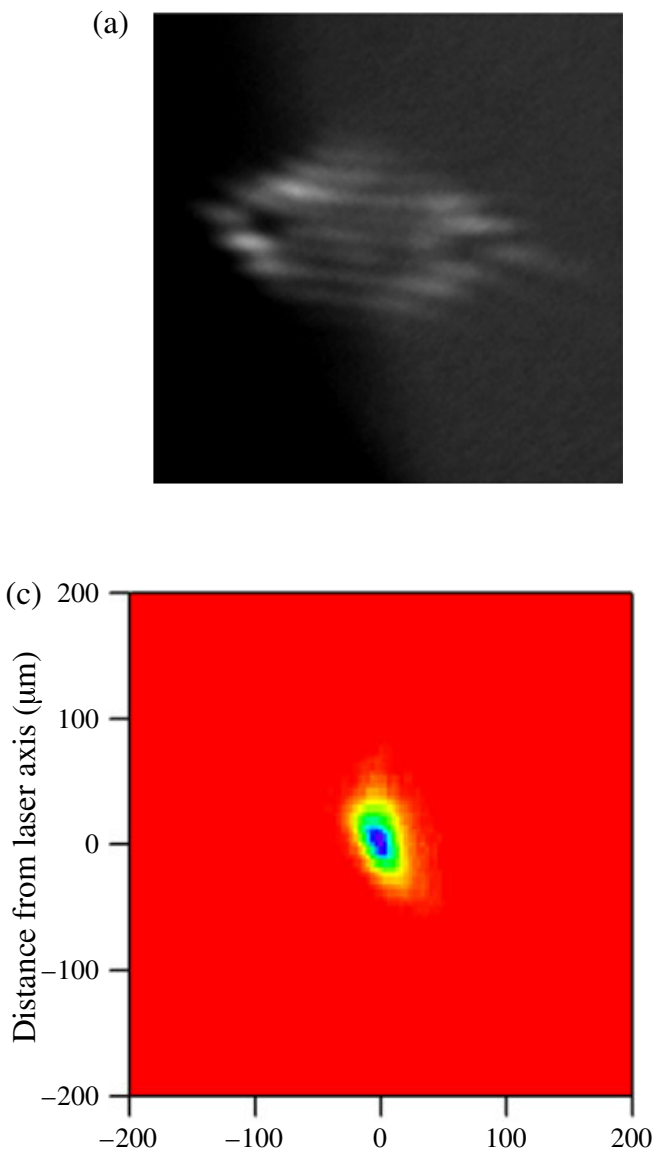

Distance from laser axis $(\mu \mathrm{m})$ show the quantitative improvement in focusability in moving from the use of the flat mirror to the deformable mirror.

To see the image on higher-energy shots to target, a combination of X-ray pinhole imaging and optical imaging of the plasma self-emission was used. Figure 6(c) shows the time-integrated X-ray emission from the focal spot, recorded by a pinhole camera on an image plate. The deconvolved spot size is $16 \mu \mathrm{m}$ FWHM with a limiting resolution of $15 \mu \mathrm{m}$. The streaked optical transition radiation (OTR) emission gives a central image size of $\sim 8 \mu \mathrm{m}$ FWHM (shown in Figure 6(d)) on a 100 TW shot, which gives an irradiance on target of $2 \times 10^{20} \mathrm{~W} \mathrm{~cm}^{-2}$.

\subsection{Astra-Gemini ultra-high-power delivery}

The Astra-Gemini system has a MOPA architecture. The seed pulses are generated from an ultra-short-pulse oscillator that provides low-energy, high-quality pulses of around $12 \mathrm{fs}$ duration. These are stretched to $\sim 7 \mathrm{ps}$ in a glass block before being amplified to millijoule energies in a $\mathrm{kHz}$ repetition rate preamplifier. Individual pulses are then selected by a (b)

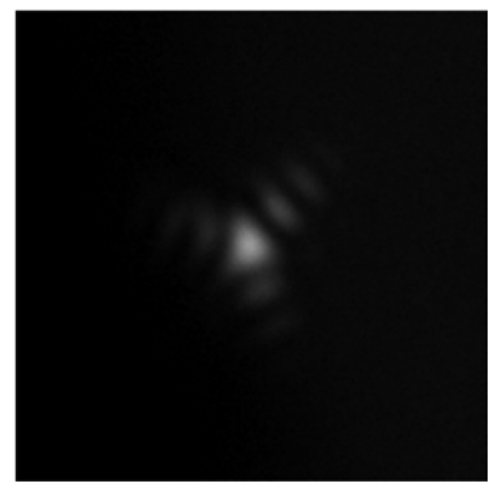

(d)

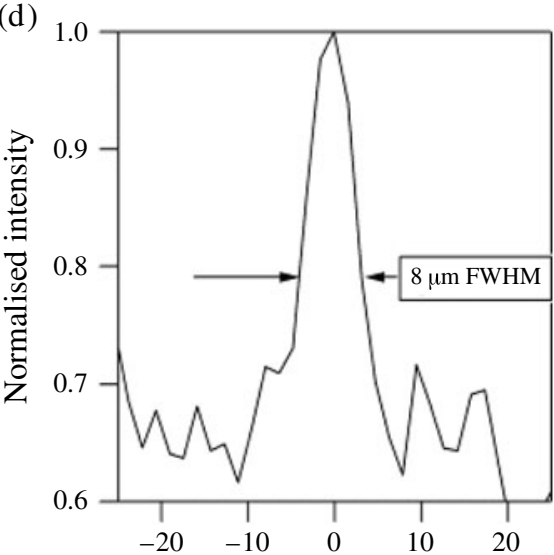

Distance at target $(\mu \mathrm{m})$

Figure 6. (a) Orion uncorrected petawatt output focal profile. (b) Orion corrected petawatt output focal profile. (c) Soft x-ray emission from an Orion target shot demonstrating an $\sim 15 \mu \mathrm{m}$ X-ray spot size. (d) Optical self-emission from an Orion target shot indicating a focal spot profile of $\sim 8 \mu \mathrm{m}$. 


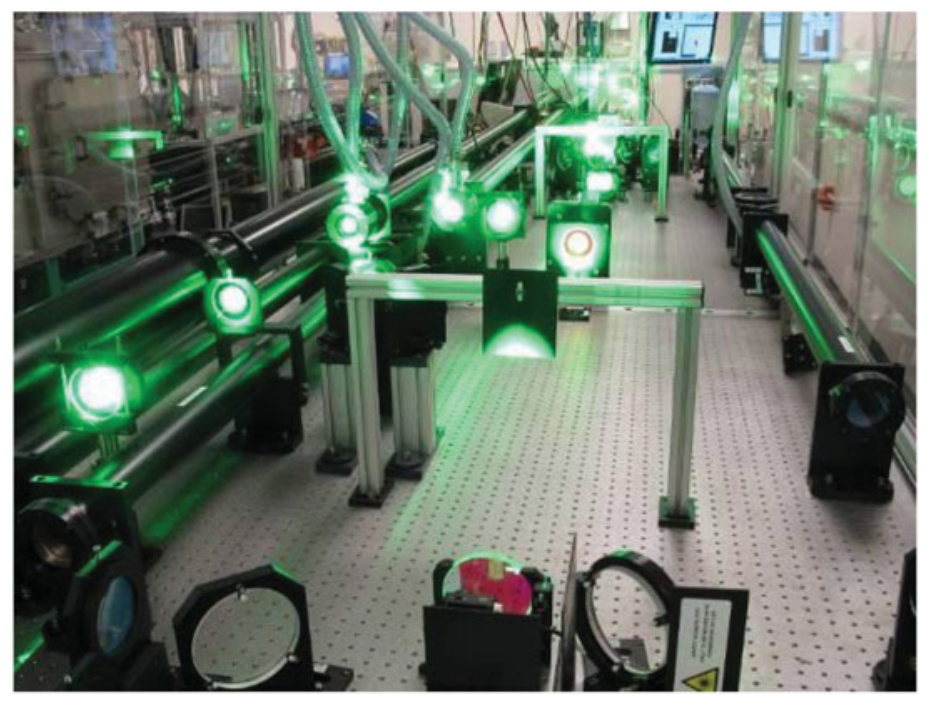

Figure 7. Astra-Gemini laser system.

fast Pockels cell at a repetition rate of $10 \mathrm{~Hz}$. A much greater stretch is then applied, using a standard grating-based pulse stretcher. The pulses are stretched to $\sim 1$ ns and then amplified in a series of Ti:Sapphire crystals that are pumped by pulses of green light from another laser. The infrared beam is sent through each crystal several times to increase efficiency. As the energy in the beam increases, both the beam size and the crystals are made larger in successive amplifiers to keep the intensity of the light below the level where damage will occur.

The Astra-Gemini output amplifiers (see Figure 7) each have a Ti:Sapphire crystal $90 \mathrm{~mm}$ in diameter and $25 \mathrm{~mm}$ thick. The design of the amplifiers calls for a small-signal gain of around 4.2 per pass, to achieve the design output energy of $25 \mathrm{~J}$. Modelling of the performance showed that this output could be achieved with a total of around $60 \mathrm{~J}$ of pump energy in a $50 \mathrm{~mm}$ diameter beam, while keeping the energy density on the crystal at a safe level. These final amplifiers have a shot rate of one shot every $20 \mathrm{~s}$.

Laser output energies of $25 \mathrm{~J}$ are delivered with around $60 \mathrm{~J}$ of pump energy, in good agreement with the modelling. At this energy, assuming a transmission efficiency of $60 \%$ in the compressor (which would be lower than average) the energy reaching the target area is $15 \mathrm{~J}$.

The main optics of the compressor are the two gratings and a plane mirror. The smaller grating is $320 \mathrm{~mm}$ by $205 \mathrm{~mm}$, and the larger is $265 \mathrm{~mm}$ by $420 \mathrm{~mm}$. Both are holographically generated with 1480 grooves per millimetre, and coated with gold. The plane mirror is coated with protected silver for increased reflectivity and damage resistance. The output bandwidth of Astra-Gemini is $\sim 35 \mathrm{~nm}$, which overfills the height of the second grating after dispersion by the first grating. The compressor is therefore operated in double pass, so the spatial dispersion of the beam in the first pass is reversed in the second.
The compressed pulse is steered to the final mirror, which sends the beam downwards into a vacuum pipe connected to the interaction chamber. Each beam will deliver $15 \mathrm{~J}$ to target in a pulse of $30 \mathrm{fs}$ (i.e., a peak power of $0.5 \mathrm{PW}$ ). In the Gemini target chamber, shown in Figure 8, adaptive optics allows the wavefront to be controlled before the beams are focused with parabolic mirrors; with a focal spot as small as two microns, intensities up to $10^{22} \mathrm{~W} \mathrm{~cm}^{-2}$ can be achieved on target.

\section{3. $2 \omega$ operation}

The first experiments to demonstrate the feasibility of contrast enhancement using second harmonic generation (SHG) were conducted at small aperture and low energy $(\sim 1 \mathrm{~J})$ at the Centre for Ultrafast Optical Science, University of Michigan by Gerard Mourou's group ${ }^{[12,13]}$. The Chien research describes SHG experiments using 500 fs laser pulses at the joule level, demonstrating efficiencies of $70 \%-$ $80 \%$ at intensities of $400 \mathrm{GW} \mathrm{cm}^{-2}$ from a Type I KDP crystal. The Queneuille work uses SHG on a terawatt laser system to demonstrate an intensity contrast of $10^{9}$, making possible, for the first time, the study of laser-matter interactions in the ultra-high-intensity, high-contrast regime.

First large aperture $(100 \mathrm{~mm})$ trials were carried out on the Vulcan facility ${ }^{[14,15]}$. These experiments used a picosecond pulse comparing the use of $2 \mathrm{~mm}$ thick and $4 \mathrm{~mm}$ thick Type I KDP crystals. Type I doubling is ideal as it produces the $1 \omega$ and $2 \omega$ outputs orthogonally polarized such that they can be separated. The experiments showed (see Figure 9) that a $4 \mathrm{~mm}$ thick crystal gives optimum conversion efficiency at an irradiance of $150 \mathrm{GW} \mathrm{cm}^{-2}$ and the optimum conversion efficiency for a $2 \mathrm{~mm}$ thick crystal is at an intensity greater than $250 \mathrm{GW} \mathrm{cm}^{-2}$ with efficiencies of $\sim 60 \%$. These experiments also demonstrated that at higher energies the 


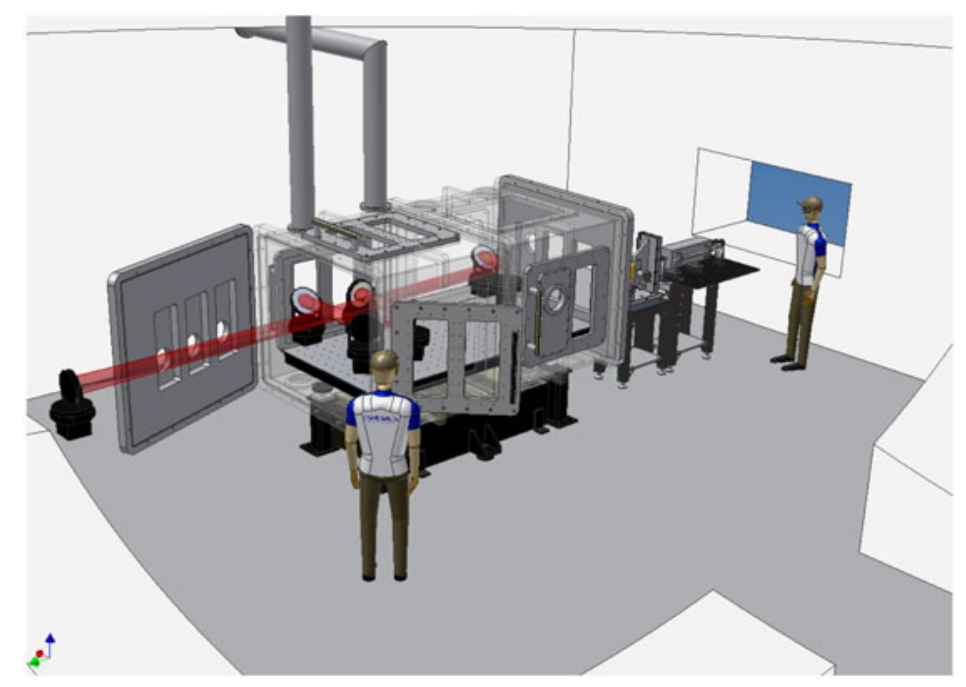

Figure 8. Astra-Gemini target chamber.

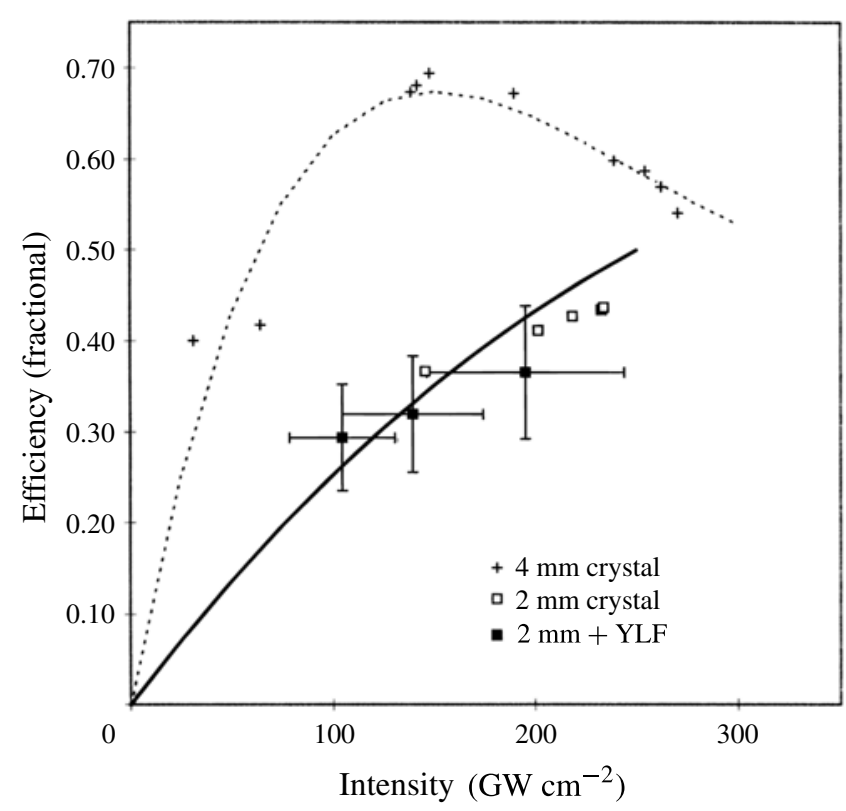

Figure 9. Comparison of the frequency conversion efficiencies of a 2 and a 4 mm Type I KDP frequency-doubling crystal.

focal spot would break up, drastically reducing the delivered focused intensity.

The frequency-doubling experiments at large aperture on Vulcan showed that a $2 \mathrm{~mm}$ Type I KDP crystal was the optimal choice. It was anticipated, and subsequently demonstrated, that $10^{19} \mathrm{~W} \mathrm{~cm}^{-2}$ could be delivered to target in a frequency-doubled beam, with a much enhanced contrast ratio, opening up new plasma physics experiments.

The first frequency-doubling contrast enhancement experiments to be carried out at AWE were conducted on the HELEN facility in 2006 using $200 \mathrm{~mm}$ diameter, $2 \mathrm{~mm}$ thick Type I KDP. Although no quantitative contrast measurements were made, experimental data from buried-layer

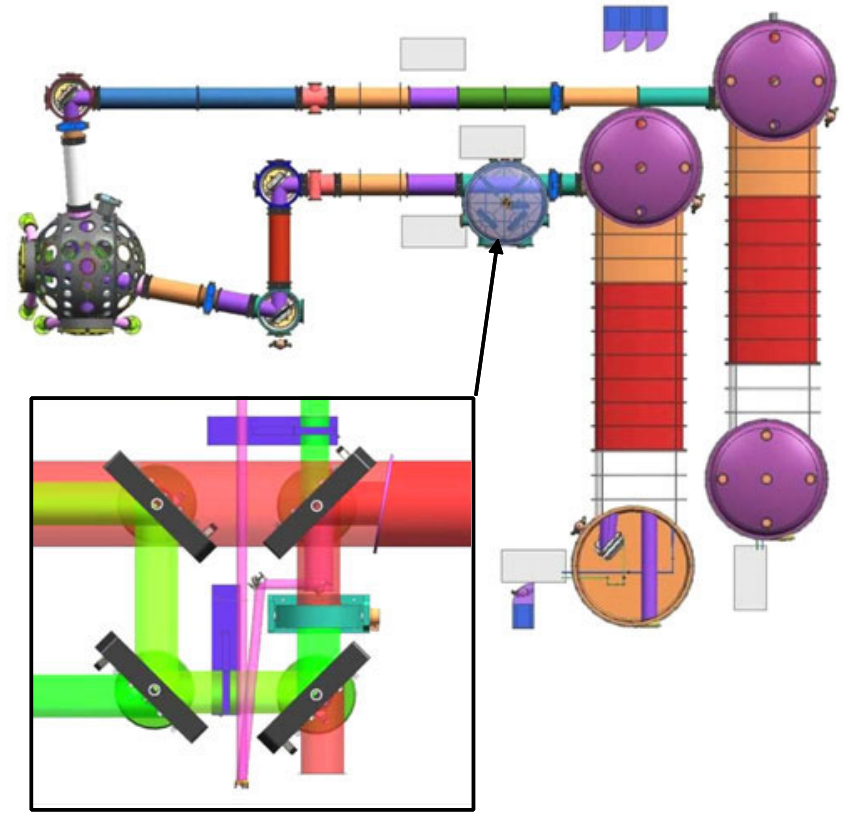

Figure 10. Schematic of the second harmonic option on Orion.

experiments suggests a greatly reduced pre-pulse ${ }^{[16]}$. Conversion efficiencies of approximately $70 \%$ were achieved for intensities of over $100 \mathrm{GW} \mathrm{cm}{ }^{-2}$ at pulse durations of 0.5 and $2 \mathrm{ps}$, with delivered target intensities of $\sim 10^{19} \mathrm{~W} \mathrm{~cm}^{-2}$.

Following the early HELEN experiments, Orion was designed from the outset to include the option of frequency doubling on one of the short-pulse petawatt beamlines. The maximum aperture crystal that could be obtained commercially was $300 \mathrm{~mm}$ with $3 \mathrm{~mm}$ thick Type I KDP. As the output of the Orion petawatt beamline is $\sim 600 \mathrm{~mm}$ this meant that the beam diameter had to be reduced by a factor of two. A schematic of the second harmonic option as installed on Orion is shown in Figure 10. 


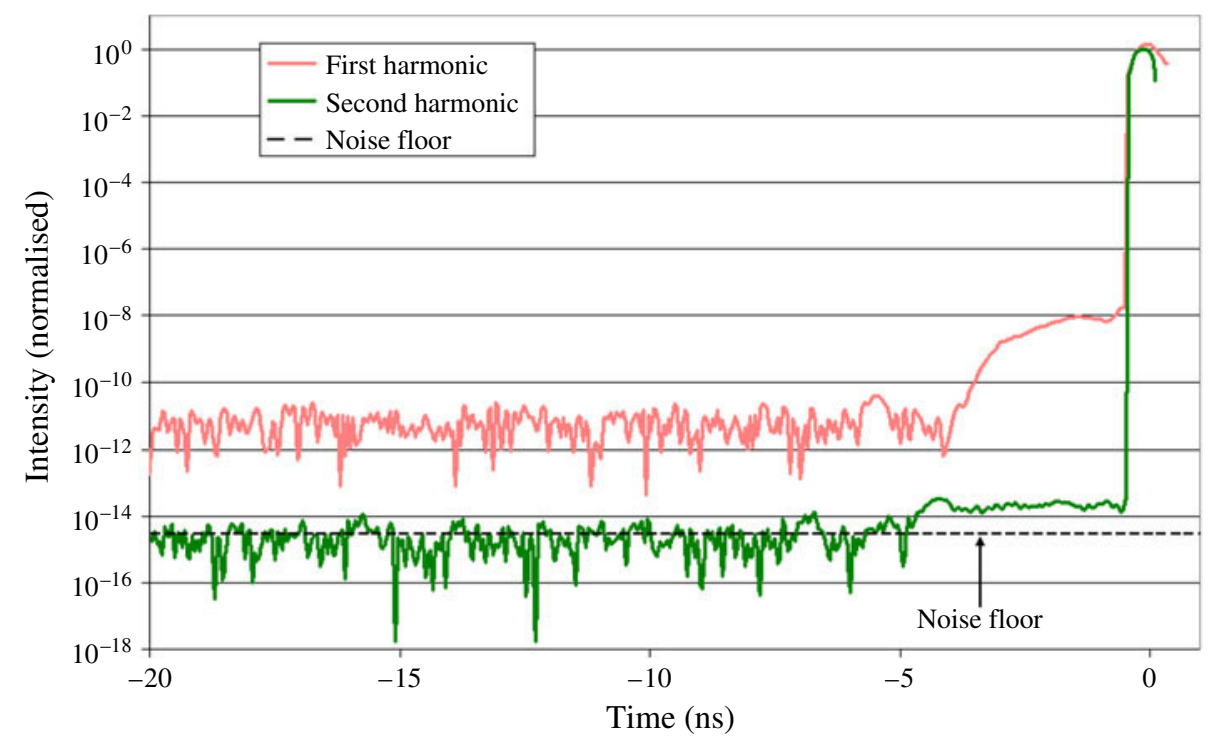

Figure 11. Contrast measurements on Orion of the fundamental and second harmonic.

Figure 10 shows the two Orion short-pulse compressor chambers on the left-hand side. Both of these are $20 \mathrm{~m}$ in length with a large aperture grating housed in each of the end chambers. The second harmonic conversion option is housed in one of these beamlines. The detail within this chamber is shown in the expanded square image. The beam is apertured down as it enters the $2 \omega$ chamber on the right-hand side. A mirror then directs it in to the $320 \mathrm{~mm}$ aperture crystal. There follow three $2 \omega$ reflective mirrors and a parabola which reject the residual $1053 \mathrm{~nm}$ light and propagate only the $527 \mathrm{~nm}$, frequency-doubled light to target. This option delivers $>100 \mathrm{~J}$ at the second harmonic (200 TW) with a $70 \%$ conversion efficiency. This can be focused down to a spot delivering $\sim 10^{20} \mathrm{~W} \mathrm{~cm}^{-2[17]}$.

The temporal contrast after compression was measured in the first harmonic from a leak through from the first mirror after the compressor. In the second harmonic it is measured from a leak through the second mirror after the frequency-doubling crystal. By concatenating photodiode (Newport 818-21A) traces from multiple shots with different attenuation levels, the temporal contrast over a large dynamic range can be measured, as shown in Figure 11. A pedestal shoulder is observed on the infrared pulse at $\sim 10^{-8}$, about 3 ns before the main pulse, which is due to parametric fluorescence in the OPCPA. When the frequency-doubling option is used, a large improvement is observed, generating a contrast to target of $\sim 10^{-14}$.

In both measurements, biased silicon photodiodes were used. To achieve sufficient dynamic range the photodiodes required extensive baffling, spectral filtering, and shielding to prevent any stray reflections or scattered light within the diagnostics enclosure swamping the signal. These measurements were all performed with no protection in front of the photodiodes (water cells, etc.), which shortened their life and that of some of the optics in the diagnostics line significantly.

\section{High-contrast front-end}

The concept of using an OPCPA system as a seed for the front-end of a high-power $\mathrm{Nd}$ :glass laser system was first proposed in Ref. [10]. This allowed an ultra-short pulse to be amplified in a broad-bandwidth preamplifier before injection in the larger aperture Nd:Glass chain. The first OPCPA front-end system became operational on the Vulcan facility in $1998^{[18,19]}$. In subsequent years, many facilities have implemented these front-end systems [20-22]. The OPCPA technique is an excellent broad-bandwidth amplifier (from nanojoule levels to tens of millijoules), producing fluorescence which generates a nanosecond pre-pulse shoulder on the pulse which gets amplified through to target. This fluorescence produces a contrast limit of $\sim 10^{-8}$ (this contrast level is still far better than that generated when using a traditional Nd:glass regenerative amplifier, where levels of $\sim 10^{-3}$ have been measured ${ }^{[23]}$, or when using multiple Nd:glass phosphate amplifiers, where levels of $\sim 10^{-6}$ have been measured ${ }^{[24]}$ ). This fluorescence is present only when the nonlinear crystal is pumped; therefore, by going to picosecond pumping it is possible to enhance the contrast.

Itantini et al., at the University of Michigan first introduced the concept of injecting an ultra-clean picosecond pulse to achieve good amplified contrast ${ }^{[25]}$ where an ultrashort-pulse preamplifier was used for the first three orders of magnitude gain in a Ti:Sapphire system. Other contrast enhancement techniques have been used based on double optical $\mathrm{CPA}^{[26]}$, where the pulse is stretched, amplified and 


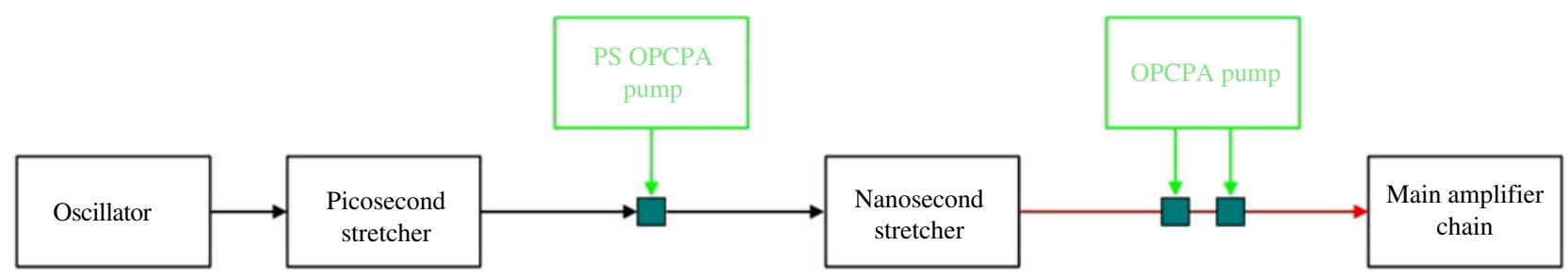

Figure 12. Scheme to introduce an additional picosecond stretcher.

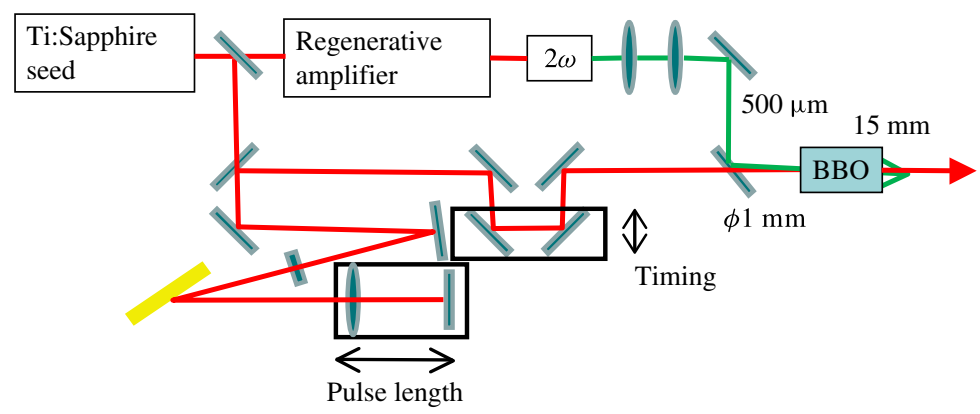

Figure 13. Schematic of the picosecond stretcher used on Vulcan for contrast enhancement.

compressed, then passed through a nonlinear filter before being stretched again and passed to the main beamlines. A range of nonlinear filters are used, depending on the pulse duration, energy and wavelength of the system, these include hollow waveguides, birefringence, cross polarization and low-gain OPA ${ }^{[27-30]}$. These nonlinear techniques suffer from a degree of complexity in their set-up and operation as they are very sensitive to pulse-to-pulse instabilities. They can also have very low throughput efficiencies, $<10 \%$.

An alternative approach first developed for Omega $\mathrm{EP}^{[31]}$ was used on the Vulcan facility ${ }^{[32]}$, where a single stage of amplification was introduced with the pulse a few picoseconds before the main stretcher, a schematic of which is shown in Figure 12. This technique reduces the amount of nanosecond gain by using a picosecond OPCPA stage.

The initial seed pulses of 200 fs duration are generated from a commercial Ti:Sapphire oscillator. These pulses are then stretched to $3 \mathrm{ps}$ and amplified in a single-stage OPCPA to take the pulses from $10 \mathrm{pJ}$ to $70 \mu \mathrm{J}$. A schematic of the picosecond stretch and amplification scheme is shown in Figure 13.

In this scheme, a common seed is used for both the signal and pump pulses, thus ensuring they are optically synchronized. The pump seed pulse is fed into the regenerative amplifier and then frequency-doubled to generate pulses of $500 \mu \mathrm{J}$ to pump the BBO crystal. The regenerative amplifier uses $\mathrm{Nd}$ :YLF as the gain medium, producing gain narrowing as the pulse is amplified, increasing the pulse duration to $\sim 10$ ps. A stretcher in the signal beam enables pulse length matching between the two pulses and a dog-leg timing adjuster ensures synchronization.
Following picosecond amplification, the pulse is stretched to the nanosecond regime before being amplified in two further OPCPA stages and then injected into the Nd:Glass amplifiers. This increased seed energy has led to an improvement of the nanosecond ASE contrast intensity to $10^{10}$ of the main pulse, without degrading the output performance of the Vulcan Petawatt system.

Following the successful demonstration of the highcontrast front-end system on Vulcan, a very similar configuration was installed on the Orion facility. This also demonstrated a nanosecond ASE contrast improvement by $\sim 2$ orders of magnitude. Figure 14 shows the initial pulse (in red), with a 3 ns shoulder, and the pulse following the deployment of the high-contrast front-end (in blue).

\section{Optics fidelity}

Typically, in Ti:Sapphire-based petawatt class laser systems there is a 'coherent' contrast feature that frequently appears as an exponentially-rising pedestal within a few tens of picoseconds of the compressed pulse. Contrast measurements ${ }^{[33]}$ were made with several optical configurations of the pulse stretcher, in which different components were eliminated or replaced, thus allowing us to distinguish the contribution to the coherent pedestal from different optics. These results show that scatter from mirrors and dispersion by a prism with polished surfaces did not make a measurable contribution to the coherent pedestal, whereas scatter from the diffraction gratings in the pulse stretcher gave its main contribution.

New higher-quality gratings were installed in the AstraGemini pulse stretcher. The new gratings are etched directly 


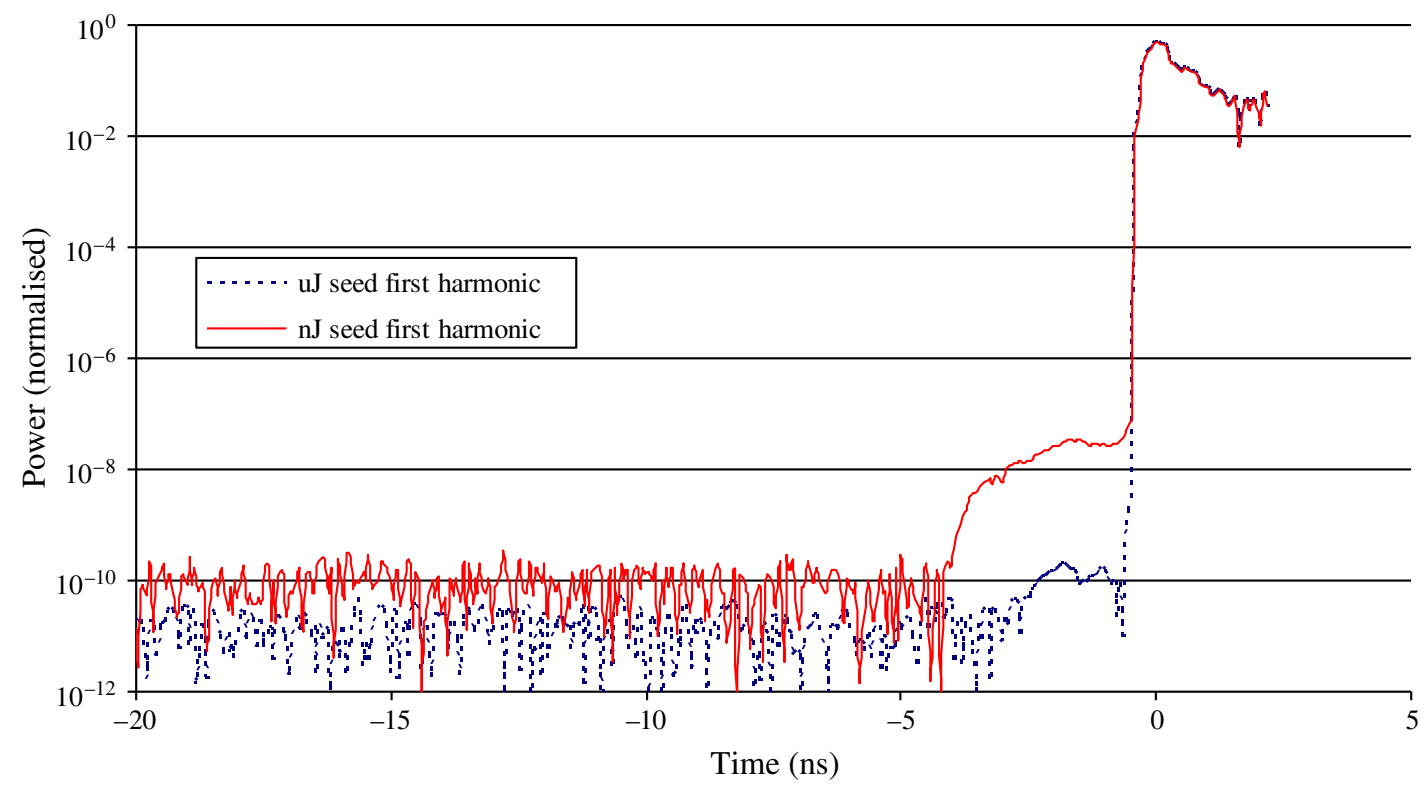

Figure 14. Measurement of contrast improvement on the Orion facility from introducing the high-contrast front-end system.

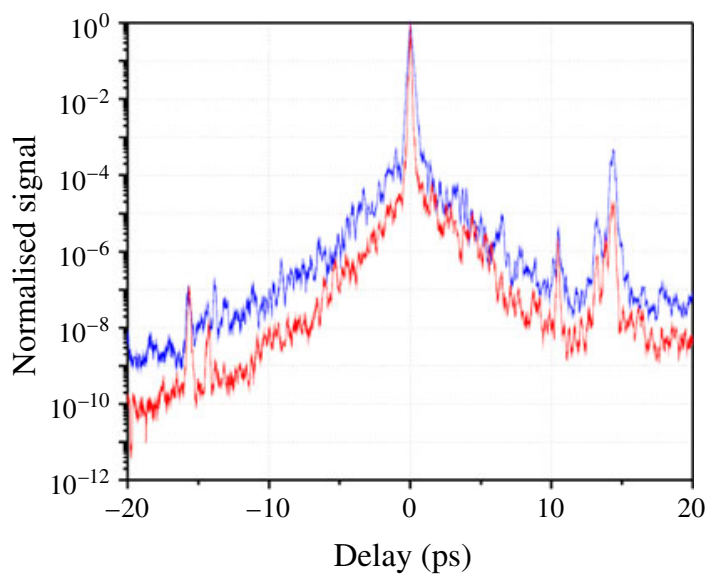

Figure 15. Contrast measurements on the Astra-Gemini system with the original gratings in blue and the replacement gratings in red.

into the fused silica substrates, rather than being formed in a photoresist layer. The contrast was measured both before and after the stretcher gratings were replaced and the results shown in Figure 15. The red line shows the initial contrast measurement and the blue line shows the contrast following grating replacement. This demonstrates an orderof-magnitude reduction in the intensity of the coherent pedestal, giving a significant improvement in the overall contrast of the compressed pulse from the laser.

The etching process may reduce the roughness of the grating surface, giving lower scatter and leading to the observed improvement in contrast. In the ten or more years since the original gratings were made, the technology of grating production has advanced in response to the requirements for high-energy CPA lasers such that gratings made today undoubtedly have better quality.

\section{Plasma mirror operation}

The use of plasma mirrors close to the target interaction is a technique that can be used to suppress unwanted prepulses $^{[34-36]}$ and has been demonstrated to deliver a contrast enhancement of over two orders of magnitude using a single mirror $^{[35,37]}$. The operation of a plasma mirror is shown in the visualization in Figure 16. At a certain intensity a laser pulse will form a plasma on the surface of a substrate. Any pre-pulse up to the point of plasma formation will be transmitted through the substrate. At times soon after the formation of the plasma there is no plasma expansion and the rest of the pulse will be reflected with relatively high efficiencies, thereby enhancing the pulse contrast.

In an experiment at the $\mathrm{CLF}^{[38]}$ the specular reflectivity of plasma mirrors formed by sub-picosecond pulses from a Ti:Sapphire laser was measured for different angles of incidence and for two different pulse lengths as a function of the laser intensity. Laser pulses with energies up to $250 \mathrm{~mJ}$ and pulse durations of 90 and 500 fs were focused onto a flat fused silica substrate. A focusing optic with a large Fnumber (F/17) and focal length of $1016 \mathrm{~mm}$ was used to avoid averaging over a large range of different angles of incidence. The contrast of the pulse was measured at a level of $<10^{-8} \times E_{\text {main }}$. The specular reflectivity was measured for intensities on target between $10^{12}$ and $4 \times 10^{17} \mathrm{~W} \mathrm{~cm}^{-2}$.

The target was moved after each shot. The intensity on target was varied either by moving the target out of focus (z-scan) or by decreasing the laser energy with a constant focal spot size. A typical result from the paper, as shown in Figure 17, demonstrates that reflectivities of over $80 \%$ can be achieved at low angles ( $6^{\circ}$ in this case) and at intensities on the substrate of $10^{15}-10^{16} \mathrm{~W} \mathrm{~cm}^{-2}$. For intensities above 


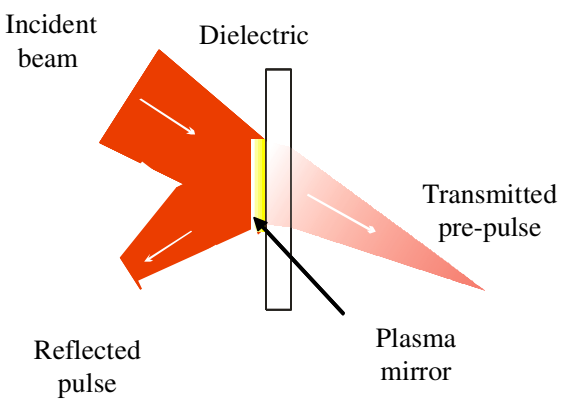

Figure 16. Cartoon of plasma mirror operation.

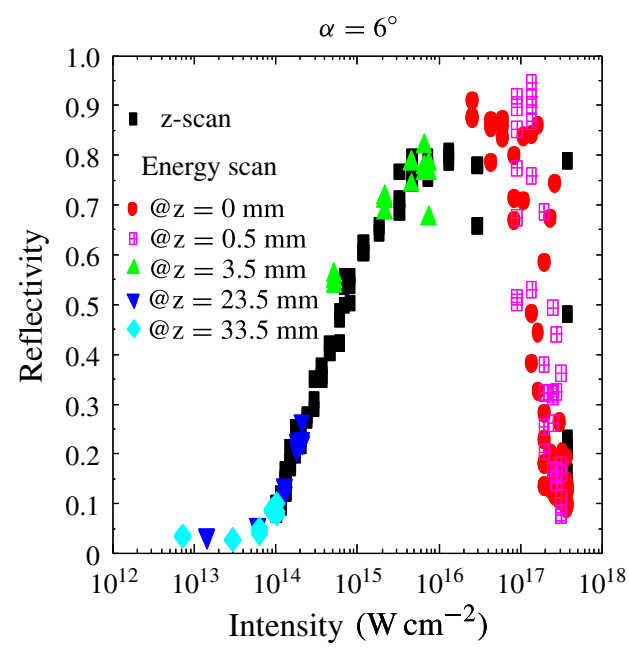

Figure 17. Typical data from the Ziener plasma mirror experiments.

mid $10^{16} \mathrm{~W} \mathrm{~cm}^{-2}$ the specular reflectivity decreases again, to values of only a few percent. The drop-off in specular reflectivity has been attributed to an increase in non-specular reflectivity (scatter, absorption or diffuse reflectivity at the plasma surface).

The relative merits of using 's' or 'p' polarization have been discussed and verified experimentally in many individual publications. As we often use plasma mirrors at large angles, it is observed that good reflectivities can be obtained when using the 's' polarization but efficiencies drop off when using the ' $p$ ' polarization, due to higher absorption of the laser light. A more complete characterization of plasma mirrors, both experimentally and theoretically, can be found in the paper by researchers at the CEA Saclay ${ }^{[37]}$.

During the past few years, the routine use of plasma mirrors on Astra-Gemini has moved from being a research project to a properly engineered capability. A system has been installed in the Astra-Gemini target chamber consisting of a $10 \times 2 \mathrm{~cm}$ plasma mirror capable of delivering 100 shots driven by a motorized stage before needing to be changed, as shown in Figure 18.

The system is a folded geometry for compactness and consists of two parabolic mirrors which allow an enhanced contrast collimated beam to be delivered to the final focusing

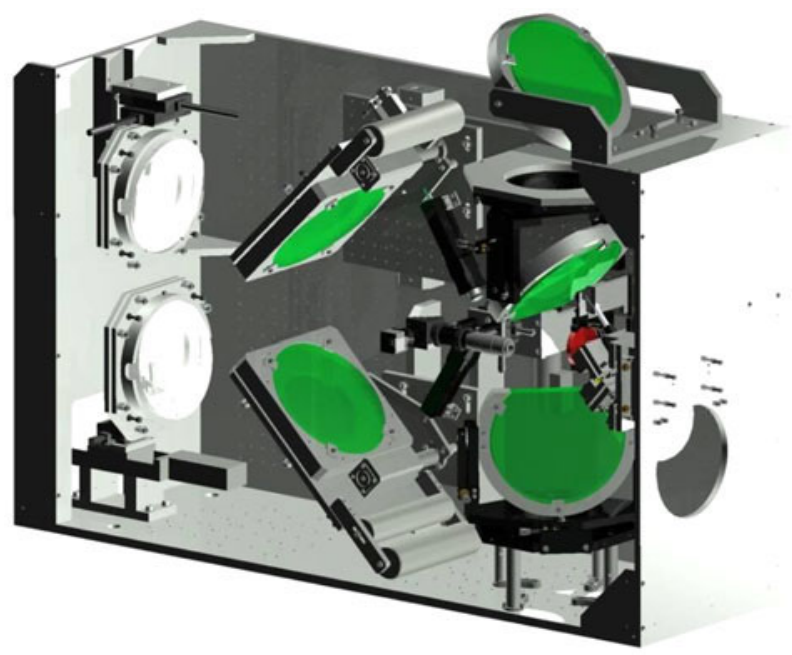

Figure 18. Double plasma mirror system on Astra-Gemini.

optic within the target chamber. A pair of by-pass optics can be inserted into the system for alignment and experimental set-up.

The contrast improvement from employing the double plasma mirror could not be absolutely measured owing to the use of an autocorrelator which had a dynamic range limit of $\sim 10^{9}$. The real contrast is believed to be $10^{-12}$ at $1 \mathrm{~ns}$, giving a pre-pulse free relativistic interaction to within 2 ps of the peak of the main interaction pulse.

\section{Discussion of contrast enhancement}

The techniques discussed can be used together to enhance the contrast further. For instance, the $2 \omega$ contrast enhancement has been used on Orion with the high-contrast front-end. A contrast ratio of $10^{-17}-10^{-19}$ is anticipated, but at the time of writing this had not been measured. The plasma mirror technique has also been used on Vulcan in tandem with its high-contrast front-end operation.

In the early 1990s, as the first CPA systems were coming on line, contrast was not the highest priority and $10^{-6}$ was seen as the state of the art. When this was first aired, a useful visualization was that this ratio was the same as the ratio of the size of a grain of sand to the height of the Eiffel tower. Having recently measured contrast ratios of $10^{-14}$ it has been pointed out that this is now the same ratio as the same grain of sand but now to the distance to the Sun (with $10^{-19}$ being the ratio of a grain of sand to the distance to Alpha Centuari!!!).

\section{Conclusion}

In the twenty years or so of development of ultra-high-power laser facilities to the petawatt level there have been dramatic 
improvements in the pulse fidelity delivered to target, critical in achieving the highest focused intensities and the highest possible contrast. This paper has summarized how these developments have been implemented on the petawatt class laser facilities based in the UK which are currently open for access to the academic community: Orion at AWE, Aldermaston and Vulcan \& Astra-Gemini at the CLF, STFC RAL.

\section{Acknowledgements}

Thanks to the Orion, Astra-Gemini and Vulcan teams who provided input for this paper and in particular to Steve Hawkes, Chris Hooker, Nick Hopps, Ian Musgrave, Rajeev Pattathil and Trevor Winstone for assistance in putting it all together.

\section{References}

1. N. Hopps, C. Danson, S. Duffield, D. Egan, S. Elsmere, M. Girling, E. Harvey, D. Hillier, M. Norman, S. Parker, P. Treadwell, D. Winter, and T. Bett, Appl. Opt. 52, 3597 (2013).

2. M. J. Norman, J. E. Andrew, T. H. Bett, R. K. Clifford, J. E. England, N. W. Hopps, K. W. Parker, K. Porter, and M. Stevenson, Appl. Opt. 41, 3497 (2002).

3. C. N. Danson, P. A. Brummitt, R. J. Clarke, J. L. Collier, B. Fell, A. J. Frackiewicz, S. Hancock, S. Hawkes, C. HernandezGomez, P. Holligan, M. H. R. Hutchinson, A. Kidd, W. J. Lester, I. O. Musgrave, D. Neely, D. R. Neville, P. A. Norreys, D. A. Pepler, C. J. Reason, W. Shaikh, T. B. Winstone, R. W. W. Wyatt, and B. E. Wyborn, IAEA J. Nucl. Fusion 44, S239 (2004).

4. C. Hernandez-Gomez, P. A. Brummitt, D. J. Canny, R. J. Clarke, J. Collier, C. N. Danson, A. M. Dunne, B. Fell, A. J. Frackiewicz, S. Hancock, S. Hawkes, R. Heathcote, P. Holligan, M. H. R. Hutchinson, A. Kidd, W. J. Lester, I. O. Musgrave, D. Neely, D. R. Neville, P. A. Norreys, D. A. Pepler, C. J. Reason, W. Shaikh, T. B. Winstone, and B. E. Wyborn, J. Phys. IV France 133, 555 (2006).

5. I. Musgrave, A. Boyle, D. Carroll, R. Clarke, R. Heathcote, M. Galimberti, J. Green, D. Neely, M. Notley, B. Parry, W. Shaikh, T. Winstone, D. Pepler, A. Kidd, C. HernandezGomez, and J. Collier, Proc. SPIE 8780, 878003 (2013).

6. C. J. Hooker, S. Blake, O. Chekhlov, R. J. Clarke, J. L. Collier, E. J. Divall, K. Ertel, P. S. Foster, S. J. Hawkes, P. Holligan, B. Landowski, B. J. Lester, D. Neely, B. Parry, R. Pattathil, M. Streeter, and B. E. Wyborn, in Proceedings of CLEO JThB2 (2008).

7. D. Strickland and G. Mourou, Opt. Commun. 56, 219 (1985).

8. M. D. Perry, D. Pennington, B. C. Stuart, G. Tietbohl, J. A. Britten, C. Brown, S. Herman, B. Golick, M. Kartz, J. Miller, H. T. Powell, M. Vergino, and V. Yanovsky, Opt. Lett. 24, 160 (1999).

9. A. Dubietis, G. Jonusauskas, and A. Piskarskas, Opt. Commun. 88, 437 (1992).

10. I. N. Ross, P. Matousek, M. Towrie, A. J. Langley, and J. L. Collier, Opt. Commun. 144, 125 (1997).

11. C. N. Danson, P. A. Brummitt, R. J. Clarke, J. L. Collier, B. Fell, A. J. Frackiewicz, S. Hawkes, C. Hernandez-Gomez,
P. Holigan, M. H. R. Hutchinson, A. Kidd, W. J. Lester, I. O. Musgrave, D. Neely, D. R. Neville, P. A. Norreys, D. A. Pepler, C. J. Reason, W. Shaikh, T. B. Winstone, R. W. W. Wyatt, and B. E. Wyborn, Laser Part. Beams 23, 87 (2005).

12. C. Y. Chien, R. S. Craxton, G. Korn, J. S. Coe, J. Squier, and G. Mourou, Opt. Lett. 20, 353 (1995).

13. J. Queneuille, F. Druon, A. Maksimchuk, G. Chériaux, G. Mourou, and K. Nemoto, Opt. Lett. 25, 508 (2000).

14. D. Neely, C. N. Danson, R. Allott, F. Amiranoff, J. L. Collier, A. E. Dangor, C. B. Edwards, P. Flintoff, P. Hatton, M. Harman, M. H. R. Hutchinson, Z. Najmudin, D. A. Pepler, I. N. Ross, M. Salvati, and T. Winstone, Laser Part. Beams 17, 281 (1999).

15. D. Neely, R. M. Allott, R. L. Clarke, J. L. Collier, C. N. Danson, C. B. Edwards, C. Hernandez-Gomez, M. H. R. Hutchinson, M. Notley, D. A. Pepler, M. Randerson, I. N. Ross, J. Springall, M. Stubbs, T. Winstone, and A. E. Dangor, Laser Part. Beams 18, 405 (2000).

16. C. R. D. Brown, D. J. Hoarty, S. F. James, D. Swatton, S. J. Hughes, J. W. Morton, T. M. Guymer, M. P. Hill, D. A. Chapman, J. E. Andrew, A. J. Comley, R. Shepherd, J. Dunn, H. Chen, M. Schneider, G. Brown, P. Beiersdorfer, and J. Emig, Phys. Rev. Lett. 106, 185003 (2011).

17. D. Hillier, C. Danson, S. Duffield, D. Egan, S. Elsmere, M. Girling, E. Harvey, N. Hopps, M. Norman, S. Parker, P. Treadwell, D. Winter, and T. Bett, Appl. Opt. 52, 4258 (2013).

18. J. Collier, C. Hernandez-Gomez, I. N. Ross, P. Matousek, C. N. Danson, and J. Walczak, Appl. Opt. 38, 7486 (1999).

19. I. N. Ross, J. L. Collier, P. I. Matousek, C. N. Danson, D. Neely, R. M. Allott, D. A. Pepler, C. Hernandez-Gomez, and K. Osvay, Appl. Opt. 39, 2422 (2000).

20. V. Bagnoud, I. A. Begishev, M. J. Guardalben, J. Puth, and J. D. Zuegel, Opt. Lett. 30, 1843 (2005).

21. H. Kiriyama, M. Mori, Y. Nakai, T. Shimomura, M. Tanoue, A. Akutsu, S. Kondo, S. Kanazawa, H. Okada, T. Motomura, H. Daido, T. Kimura, and T. Tajima, Opt. Lett. 33, 645 (2008).

22. J. Schwarz, P. Rambo, M. Geissel, M. Kimmel, E. Brambrink, B. Atherton, and J. Glassman, Opt. Commun. 281, 4984 (2008).

23. Y. Kitagawa, H. Fujita, R. Kodama, H. Yoshida, S. Matsuo, T. Jitsuno, T. Kawasaki, H. Kitamura, T. Kanabe, S. Sakabe, K. Shigemori, N. Miyanaga, and Y. Izawa, IEEE J. Quantum Electron. 40, 281 (2004).

24. C. N. Danson, L. J. Barzanti, Z. Chang, A. E. Damerell, C. B. Edwards, S. Hancock, M. H. R. Hutchinson, M. H. Key, S. Luan, R. R. Mahadeo, L. P. Mercer, P. Norreys, D. A. Pepler, D. A. Rodkiss, I. N. Ross, M. A. Smith, R. A. Smith, P. Taday, W. T. Toner, K. W. M. Wigmore, T. B. Winstone, R. W. W. Wyatt, and F. Zhou, Opt. Commun. 103, 392 (1993).

25. J. Itatani, J. Faure, M. Nantel, G. Mourou, and S. Watanabe, Opt. Commun. 148, 70 (1998).

26. M. P. Kalashnikov, E. Risse, H. Schönnagel, and W. Sandner, Opt. Lett. 30, 923 (2005).

27. D. Homoelle, A. L. Gaeta, V. Yanovsky, and G. Mourou, Opt. Lett. 27, 1646 (2002).

28. A. Jullien, F. Augé-Rochereau, G. Chériaux, J. Chambaret, P. d'Oliveira, T. Auguste, and F. Falcoz, Opt. Lett. 29, 2184 (2004).

29. A. Jullien, O. Albert, F. Burgy, G. Hamoniaux, J. Rousseau, J. Chambaret, F. Augé-Rochereau, G. Chériaux, J. Etchepare, N. Minkovski, and S. M. Saltiel, Opt. Lett. 30, 920 (2005).

30. R. C. Shah, R. P. Johnson, T. Shimada, K. A. Flippo, J. C. Fernandez, and B. M. Hegelich, Opt. Lett. 34, 2273 (2009).

31. C. Dorrer, I. A. Begishev, A. V. Okishev, and J. D. Zuegel, Opt. Lett. 32, 2143 (2007). 
32. I. Musgrave, W. Shaikh, M. Galimberti, A. Boyle, C. Hernandez-Gomez, K. Lancaster, and R. Heathcote, Appl. Opt. 49, 6558 (2010).

33. C. Hooker, Y. Tang, O. Chekhlov, J. Collier, E. Divall, K. Ertel, S. Hawkes, B. Parry, and P. P. Rajeev, Opt. Express 19, 2193 (2011).

34. H. C. Kapteyn, M. M. Murnane, A. Szoke, and R. W. Falcone, Opt. Lett. 16, 490 (1993).

35. D. M. Gold, Opt. Lett. 19, 2006 (1994).
36. A. Tien, M. Nantel, G. Mourou, D. Kaplan, and M. Bouvier, Opt. Lett. 22, 1559 (1997).

37. G. Doumy, F. Quéré, O. Gobert, M. Perdrix, Ph. Martin, P. Audebert, J. C. Gauthier, J.-P. Geindre, and T. Wittmann, Phys. Rev. E 69, 026402 (2004).

38. Ch. Ziener, P. S. Foster, E. J. Divall, C. J. Hooker, M. H. R. Hutchinson, A. J. Langley, and D. Neely, J. Appl. Phys. 93, 768 (2003). 